\title{
Carisbamate (RWJ-333369)
}

\author{
Gerald P. Novak, Michael Kelley, Peter Zannikos, and Brian Klein
}

Johnson \& Johnson, Pharmaceutical Research \& Development L.L.C., Titusville, New Jersey 08560

\begin{abstract}
Summary: Carisbamate (RWJ-333369) is a novel neuromodulator, initially developed by SK Biopharmaceuticals (Fairlawn, NJ), under development by Johnson \& Johnson Pharmaceutical Research and Development (La Jolla, CA). Carisbamate displays high potency in a broad range of rodent seizure models at doses well below those that produce CNS toxicity. Its mechanism of action has not been elucidated. Acute and chronic nonclinical toxicological studies have not revealed any significant abnormalities other than dose-related CNS toxicity. It is extensively metabolized, chiefly through glucuronidation and oxidation of the aliphatic side chain. There is little evidence of
\end{abstract}

CYP metabolism. It has linear pharmacokinetics. Its clearance is increased by carbamazepine and to a lesser degree by oral contraceptives. Carisbamate slightly increases the clearance of valproic acid and lamotrigine. The most common adverse events in humans are headaches, dizziness, and somnolence, generally mild to moderate, occurring at doses of $1000 \mathrm{mg}$ /day or more. A recently completed phase 2 study for adjunctive use in partial onset seizures showed efficacy at a dose that was well tolerated. Key Words: Epilepsy, antiepileptic drug, rodent seizure models, pharmacokinetics, drug interactions.

\section{INTRODUCTION}

Carisbamate (S-2-O-carbamoyl-1-o-chlorophenyl-ethanol; FIG. 1) is a novel neuromodulator under development for the treatment of epilepsy. It has an excellent profile in preclinical models of epilepsy and has demonstrated efficacy and tolerability in a phase II clinical trial.

\section{PHARMACOLOGY}

Carisbamate was found to possess a broad spectrum of activity in rodent seizure and epilepsy models. In acute seizure models, carisbamate exhibited potent activity against seizures induced by maximal electroshock, pentylenetetrazol, bicuculline, and picrotoxin ${ }^{1}$ (TABLE 1). The $\mathrm{ED}_{50}$ values for oral administration in the maximal electroshock test were $7.7 \mathrm{mg} / \mathrm{kg}$ in mice and $4.4 \mathrm{mg} / \mathrm{kg}$ in rats. ${ }^{1}$ Carisbamate was also evaluated in the Wistar audiogenic sensitive rat and at $10 \mathrm{mg} / \mathrm{kg}$ (i.p.) significantly delayed seizure onset, and at $20 \mathrm{mg} / \mathrm{kg}$ (i.p) completely blocked all seizure-related behaviors. ${ }^{2}$ In kindling models of complex partial seizures, carisbamate effectively reduced the seizure severity in the corneal kindled rat model. ${ }^{1}$ In this model, carisbamate produced a dose-

Address correspondence and reprint requests to: G.P. Novak, MD, Johnson \& Johnson, Pharmaceutical Research \& Development L.L.C., Titusville, NJ 08560. E-mail: GNovak1@PRDUS.JNJ.COM. dependent reduction in seizure severity. At the highest dose tested (i.e., $100 \mathrm{mg} / \mathrm{kg}$, oral), the seizure score was reduced from 5 to 0 in all of the rats exposed. Carisbamate was also very effective in reducing the seizure severity and after-discharge duration ( $49.5 \%$ at $25 \mathrm{mg} / \mathrm{kg}$ i.p.) in hippocampal kindled rats. In the genetic absence epilepsy rat from Strasbourg model of absence epilepsy, the duration of spike-and-wave discharges were significantly suppressed by carisbamate at $30 \mathrm{mg} / \mathrm{kg}$ (i.p.) with complete suppression at $60 \mathrm{mg} / \mathrm{kg}$ (i.p.). ${ }^{2}$ Carisbamate was evaluated for activity against spontaneous recurrent seizures in the kainate post-status epilepticus model of temporal lobe epilepsy. At $10 \mathrm{mg} / \mathrm{kg}$ and $30 \mathrm{mg} / \mathrm{kg}$ (i.p.) carisbamate significantly reduced the frequency of spontaneous seizures $(74 \%$ reduction at $30 \mathrm{mg} / \mathrm{kg}, P<$ 0.0001). ${ }^{3}$ Furthermore, carisbamate was more effective than topiramate in completely suppressing spontaneous recurrent seizures in a larger fraction of the rats studied (i.e., seven of eight rats completely suppressed at 30 $\mathrm{mg} / \mathrm{kg}$ i.p. versus one of eight rats completely suppressed at $100 \mathrm{mg} / \mathrm{kg}$ i.p., respectively). In addition, carisbamate was evaluated for disease modification potential in the lithium-pilocarpine-induced status epilepticus model. When dosed two times daily for six days starting one hour after the onset of status epilepticus, carisbamate at $60-120 \mathrm{mg} / \mathrm{kg}$ (i.p.) reduced neuron loss and dose dependently delayed or prevented the development of spontaneous recurrent seizures. ${ }^{4}$ These results suggest 
<smiles>NC(=O)OC[C@H](O)c1ccccc1Cl</smiles>

FIG 1. Carisbamate (RWJ-333369).

that carisbamate at high doses may have disease modifying effects in the lithium-pilocarpine model. Overall, carisbamate exhibited a profile of broad-spectrum activity in rodent models of generalized, absence and partial epilepsy at nontoxic doses. The molecular actions of carisbamate that contribute to its broad-spectrum antiepileptic activity have not been elucidated and remain under investigation.

\section{NONCLINICAL TOXICOLOGY}

Carisbamate is efficacious in rodent seizure models at doses well below those that produce CNS toxicity. In rotorod and inclined screen tests in mice, and observed minimal motor impairment in rats, the single-dose oral $\mathrm{TD}_{50}$ for motor impairment was $\geq 137 \mathrm{mg} / \mathrm{kg}$, resulting in a protective index $\left(\mathrm{TD}_{50} / \mathrm{ED}_{50}\right.$ in maximal electroshock) of $\geq 15$. The maximum tolerated single dose ranged from 360 to $600 \mathrm{mg} / \mathrm{kg}$ orally in mice and rats; these doses produced decreased activity, ataxia, sedation, and prostration.

No significant organ toxicity was observed in repeated-dose oral toxicity studies in adult rats up to six months and in adult dogs up to 12 months; these studies attained $\mathrm{C}_{\max }$ and area under the curve exposures six- to ninefold and 2.5- to eightfold greater, respectively, than anticipated human therapeutic exposures. Toxicity was also evaluated in juvenile rats orally administered carisbamate from postnatal days 12 to 54 . The CNS adverse events were elicited only at doses of 80 and $160 \mathrm{mg} / \mathrm{kg}$ in a pattern similar to adults. Moreover, carisbamate treatment of juvenile rats had no effect on learning, memory, or reproductive function. Metabolism studies in rats and dogs using ${ }^{14} \mathrm{C}$-carisbamate demonstrated that these species were appropriate models for evaluating the repeated-dose toxicity of carisbamate in humans. Carisbamate tested negative for genetic toxicity in the bacterial reverse mutation test, the in vitro human lymphocyte chromosomal aberration assay, and the in vivo oral mouse bone marrow assay.

\section{PHARMACOKINETICS AND DRUG INTERACTIONS}

Oral doses of carisbamate are well absorbed ( $>95 \%$ of total dose) and maximum plasma concentrations are achieved within one to three hours in the fasted state. Total recovery in urine is $\sim 94 \%$ of the administered dose, with only $\sim 2 \%$ as unchanged carisbamate. The apparent distribution volume of carisbamate is approximately $50 \mathrm{~L}$, indicating it does not distribute extensively to peripheral tissues. Plasma protein binding is approximately $44 \%$ and concentration independent. Carisbamate is extensively metabolized, ${ }^{5}$ chiefly through O-glucuronidation ( $44 \%$ of the dose is recovered in urine as the S-glucuronide) and hydrolysis of the carbamate ester followed by oxidation of the aliphatic side chain (resulting in chloromandelic acid, chlorobenzoic acid, and chlorophenylglycine, totaling $36 \%$ of the dose). Chiral inversion to form the R-enantiomer followed by O-glucuronidation $(11 \%)$ and hydroxylation of the aromatic ring followed by sulfation (5\%) are minor routes of metabolism. With the use of very sensitive liquid chromatography-mass spectrometry-mass spectrometry techniques, only traces of aromatic (pre) mercapturic acid conjugates were detected in urine (each $<0.3 \%$ of the dose), suggesting a low potential for reactive metabolite formation.

After single doses of $100 \mathrm{mg}$ to $1500 \mathrm{mg}$ and repetitive (q12h) doses of 100-750 mg, carisbamate had linear

TABLE 1. Anticonvulsant Profile Comparison in Acute Seizure Models

\begin{tabular}{lcccr}
\hline & \multicolumn{4}{c}{ Mouse $\mathrm{ED}_{50}(\mathrm{mg} / \mathrm{kg}$ i.p. $)$} \\
\cline { 2 - 5 } & MES & $\mathrm{scPTZ}$ & $\mathrm{sc} \mathrm{BIC}$ & sc PIC \\
\hline Carisbamate & 7.9 & 20.4 & $17^{*}$ & 11.6 \\
Topiramate & 33.0 & $>800$ & $>500$ & $>500$ \\
Valproic acid & 263 & 220 & 589.0 & 270.0 \\
Phenytoin & 5.6 & $>50.0$ & $>50.0$ & $>50.0$ \\
Lamotrigine & 7.5 & $>50.0$ & $>40.0$ & $>0.0$ \\
Carbamazepine & 7.8 & 126 & $>50.0$ & 18.2 \\
Felbamate & 35.5 & 47.5 & $>500$ & $>500$ \\
Gabapentin & 78.2 & $>500$ & 4.7 & $>500$ \\
Levetiracetam & $>50$ & &
\end{tabular}

i.p. = intraperitoneal; $\mathrm{BIC}=$ bicuculline; $\mathrm{MES}=$ maximal electroshock; PIC $=$ picrotoxin; PTZ $=$ pentylenetetrazol; sc $=$ subcutaneous.

* Clonic seizures partially inhibited. 
pharmacokinetics. ${ }^{6}$ Carisbamate has a low oral (metabolic) clearance $(\mathrm{CL} / \mathrm{F})$ of $3.4-4.2 \mathrm{~L} / \mathrm{h}$, equaling $<5 \%$ of liver blood flow. Thus, orally administered carisbamate is not susceptible to hepatic first-pass effect. The 12-hour half-life will enable twice a day dosing with an immediate-release oral formulation. ${ }^{6}$ Steady-state conditions are achieved within three days, in agreement with the known half-life of about 12 hours. ${ }^{6}$ Steady-state plasma concentrations increased by a factor of 2 (compared with single dose) when given two times daily. Consistent pharmacokinetics after single and repeat administration suggests carisbamate does not induce or inhibit its own metabolism.

Carisbamate exhibits a low propensity to alter the pharmacokinetics of coadministered drugs. Administration of $500 \mathrm{mg} /$ day or $1000 \mathrm{mg} /$ day carisbamate to healthy subjects had minimal impact on the activity of the CYP2C9, CYP2D6, and CYP3A4 enzymes, as indexed by the clearance of probe substrates of these enzymes (tolbutamide, desipramine, and midazolam, respectively). These regimens of carisbamate produced a modest and clinically insignificant reduction of valproic acid and lamotrigine (approximately $20 \%^{7}$ ); no clinically significant changes in the pharmacokinetics of carbamazepine or an oral contraceptive (Ortho-Novum, 1 of 35; [Ortho-McNeil Pharmaceutical, Inc., Raritan, NJ], containing $0.035 \mathrm{mg}$ ethinyl estradiol and $1 \mathrm{mg}$ norethindrone) were produced. Coadministration of carbamazepine $(600 \mathrm{mg} /$ day $)$ reduced the concentrations of carisbamate by $36 \%,{ }^{8}$ whereas lamotrigine $(100 \mathrm{mg} /$ day $)$ and valproic acid (1000 mg/day) had no clinically significant impact. ${ }^{7}$ Concentrations of carisbamate were reduced by $20 \%$ to $30 \%$ with concomitant administration of the oral contraceptive. The effects of carbamazepine and oral contraceptives are attributed to induction of carisbamate glucuronidation.

\section{EFFICACY DATA}

A nonrandomized, single-blind, placebo-controlled study in subjects with photosensitive epilepsy assessed the effect of carisbamate on the photoparoxysmal response. Subjects received a single oral dose of placebo on the mornings of day 1 and day 3 and a single dose of carisbamate on the morning of day 2 , after a descending algorithm ranging from 1000 to $250 \mathrm{mg}$. Photosensitivity was assessed at frequencies from 2 to $60 \mathrm{~Hz}$. The paroxysmal response to intermittent photic stimulation was quantified as the number of frequencies that elicited it. Reduction or complete suppression of the photoparoxysmal response was observed in two of four evaluable subjects receiving $500 \mathrm{mg}$ (including one with complete suppression), three of four evaluable subjects receiving $750 \mathrm{mg}$, and in all five subjects receiving $1000 \mathrm{mg}$ (including two with complete suppression).
The effect on the photosensitivity response was rapid, occurring within four hours postdose at lower doses and within two hours postdose at $1000 \mathrm{mg}$. The maximal suppression occurred close to maximal plasma levels of carisbamate. The duration of the suppression was doserelated and long lasting, with clinically significant reductions in the photosensitivity response being observed at least 32 hours after dosing with carisbamate in some subjects at the 750- and 1000-mg dose levels, despite low plasma concentrations of carisbamate.

A randomized, double-blind, placebo-controlled doseranging phase IIb study explored daily dosages of 100 $\mathrm{mg}, 300 \mathrm{mg}, 800 \mathrm{mg}$, and $1600 \mathrm{mg}$ in subjects with partial onset seizures $(n=537$ randomized $)$. Preliminary results indicate that carisbamate is efficacious in the 300 to $1600 \mathrm{mg}$ dosage range, with tolerability equivalent to placebo in $300 \mathrm{mg}$. Efficacy was apparent within the first month of treatment and remained so through the 16-week study. Daily doses of $200 \mathrm{mg}$ and $400 \mathrm{mg}$ were well tolerated in the first week, suggesting that minimal or no titration will be needed in this range.

\section{TOLERABILITY PROFILE}

The most common adverse events, encountered mainly at daily doses of $1000 \mathrm{mg}$ or more, were CNSrelated, including headache, dizziness, somnolence, and nausea. These were all generally mild and transient, or resolved with dose reduction or discontinuation. At a daily dose of $300 \mathrm{mg}$, the tolerability of carisbamate was excellent, and discontinuation because of adverse events was less than for placebo.

\section{PLANNED STUDIES}

More than $80 \%$ of subjects in this randomized trial completed the double-blind phase and entered an openlabel extension. The longest exposure in subjects with epilepsy has been more than two years. Based upon these results, carisbamate is entering phase III trials for adjunctive use in partial onset seizures.

\section{REFERENCES}

1. White HS, Srivastava A, Klein B, et al. The novel investigational neuromodulator RWJ 333369 displays a broad-spectrum anticonvulsant profile in rodent seizure and epilepsy models (abstract). Epilepsia 2006;47(suppl 4):200.

2. Nehlig A, Rigoulot M-A, Boehrer A. A new drug, RWJ-333369, displays potent antiepileptic properties in genetic models of absence and audiogenic epilepsy (abstract). Epilepsia 2005;46(suppl 8):215.

3. Grabenstatter HL, Dudek FE. The use of chronic models in antiepileptic drug discovery: The effect of RWJ-333369 on spontaneous motor seizures in rats with kainite-induced epilepsy (abstract). Epilepsia 2004;45(suppl 7):197.

4. Francois J, Ferrandon A, Koning E, Nehlig A. A new drug, RWJ333369 , protects limbic areas in the lithium-pilocarpine model (lipilo) of epilepsy and delays or prevents the occurrence of spontaneous seizures (abstract). Epilepsia 2005;46(suppl 8):269-270. 
5. Mannens GSJ, Hendricks J, Janssen C, et al. The absorption, metabolism and excretion of the novel neuromodulator RWJ-333369 in humans. Drug Metab Dispos 2006 (in press).

6. Yao C, Chien S, Doose DR, Novak J, Bialer M. Pharmacokinetics of the new antiepileptic and CNS drug RWJ-333369, following single and multiple doing to humans. Epilepsia 2006;11:1822-1829.
7. Chien S, Yao C, Mertens A. Lack of clinically significant pharmacokinetic drug interactions between RWJ-333369 and valproate or lamotrigine. Epilepsia 2005;46(suppl 8):173.

8. Chien S, Bialer M, Solanki B. Pharmacokinetic interaction study between the new antiepileptic and CNS drug RWJ-333369 and carbamazepine in healthy subjects. Epilepsia 2006;11:1830-1839. 